\title{
Laparoscopic resection of gastric wall tumor
}

\author{
Xuefei Yang, Li Jiang, Fion S. Chan \\ Department of Surgery, the University of Hong Kong-Shenzhen Hospital, the University of Hong Kong, Shenzhen 518052, China \\ Correspondence to: Fion S. Chan. Department of Surgery, the University of Hong Kong-Shenzhen Hospital, the University of Hong Kong, No. 1 \\ Haiyuan 1st Road, Futian District, Shenzhen 518052, China. Email: 4683755@qq.com.
}

\begin{abstract}
Laparoscopic resection of gastric wall tumor is commonly performed nowadays. The exact surgical procedure was decided according to the location, size and morphology of the tumor. In this video, we performed a laparoscopic resection of a $5 \mathrm{~cm}$ tumor located at distal posterior gastric wall near the greater curvature. Technical consideration was discussed.
\end{abstract}

Keywords: Laparoscopic; gastric wall tumor; resection; suture repair

Received: 07 June 2016; Accepted: 09 June 2016; Published: 15 July 2016.

doi: 10.21037 /jovs.2016.06.02

View this article at: http://dx.doi.org/10.21037/jovs.2016.06.02

\section{Introduction}

Incidental gastric wall tumor is increasingly diagnosed by upper endoscopy and imaging studies performed for other symptoms. These lesions are commonly small in size and asymptomatic. While gastrointestinal stromal tumor (GIST) is the commonest submucosal tumor located at proximal stomach, other tumors are leiomyoma, lipoma, schwannoma, etc.

Endoscopic enucleation is performed for smaller tumor in selected patients. Surgical treatment by wedge resection of stomach, partial gastrectomy or total gastrectomy is commonly required for larger lesions.

Laparoscopic resection of gastric wall tumor is advocated due to its minimally invasive nature. Comparison of laparoscopic and open resection of gastric GISTs was performed and comparable results in terms of efficacy, safety, and length of hospitalization were demonstrated in many studies (1-8). Latest meta-analysis showed that there was no observed difference in operative time, adverse events, estimated blood loss, overall survival and recurrence rates between laparoscopic and open surgical resection of gastric GISTs, supporting that laparoscopic resection is safe and effective for gastric GISTs. Laparoscopic resection was associated with a significantly shorter length of hospital stay (9). International guideline suggests that laparoscopic wedge resection can be used for tumors $\leq 5 \mathrm{~cm}(10)$.

\section{Patients}

A 57-year-old gentleman, with good past health, presented with intermittent mild upper abdominal pain for 1 year. CT scan abdomen was performed and showed a $4 \mathrm{~cm} \times 5 \mathrm{~cm}$ tumor near distal body of stomach. Upper endoscopy revealed a submucosal tumor at posterior wall of distal greater curve and it appeared to originate from muscularis propria layer on endoscopic ultrasound.

\section{Pre-operative preparation}

The patient was kept fasted for 6 hours prior to surgery. The operation was performed under general anesthesia with endotracheal intubation. A Fr 14 gastric decompression tube and urinary catheter were placed. Prophylactic cefuroxime $1.5 \mathrm{~g}$ was administrated intravenously on induction.

\section{Equipment preference card}

High definition laparoscopic video system, pneumoperitoneum system, ultrasonic dissector, laparoscopic instruments including atraumatic graspers, needle holder, scissors, 4-0 maxon suture and plastic specimen bag were prepared.

\section{Procedure}

The patient was placed in a supine position with legs 


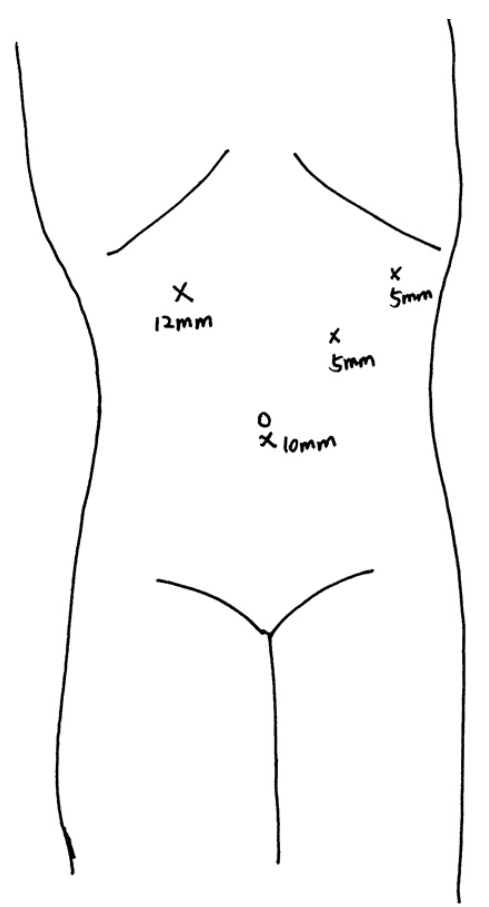

Figure 1 Sites of trocars.

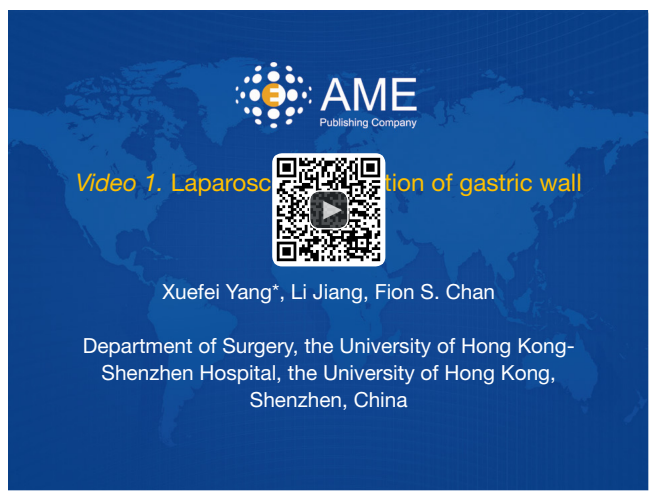

Figure 2 Laparoscopic resection of gastric wall tumor (11). Available online: http://www.asvide.com/articles/1034

separated. The chief surgeon stood between the legs. The assistant stood on the left side and the camera operator stood on the right side of the patient. A 10-mm trocar was placed in subumbilical region and pneumoperitoneum was created. The intra-abdominal pressure was maintained at $12 \mathrm{mmHg}$. The other trocar sites including a $12-\mathrm{mm}$ trocar at right upper quadrant mid-clavicular line, two 5 -mm trocars at left upper quadrant mid-clavicular line and anterior axillary line are shown in Figure 1.

Laparoscopic assessment was performed and a tumor bulge was identified in distal stomach. The greater omentum was dissected free from the greater curvature of stomach by ultrasonic dissector. The lesser sac was entered and the posterior wall of distal stomach and antrum was exposed. A 5 -cm tumor was noted arising from posterior gastric wall near greater curvature. Gastrostomy was made with ultrasonic dissector next to the tumor. Circumferential full thickness resection of gastric tumor was performed. The tumor was immediately put into a plastic specimen bag. Hemostasis of gastric wall was secured. The gastrostomy was suture repaired with 4-0 maxon in continuous manner. (Figure 2) The peritoneal cavity was cleansed with warm normal saline. The specimen was then retrieved via subumbilical trocar site after enlargement.

\section{Role of team members}

- Chief surgeon: Dr. Xuefei Yang;

- Assistant: Dr. Fion S. Chan;

- Camera operator: Dr. Li Jiang;

- Anesthetist: Dr. Xinhe Liu;

- Scrub nurse: Kaisong Hou.

\section{Post-operative management}

The patient was prescribed intravenous cefuroxime for 1 day. Gastric tube was removed on day 1 and fluid diet was commenced on day 3 after the operation. The patient was fit for discharged on day 4 with esomeprazole for 2 weeks.

Histopathological examination of resected specimen confirmed the diagnosis of schwannoma. Immunology exam showed S-100 diffusely strong positive, CD34 focal weak positive, SMA negative, Desmin negative, CD117 focal suspected positive, ki67 about 1-4\% incidental mitotic count.

\section{Tips, tricks and pitfalls}

Proper exposure of tumor is sometimes difficult for tumor located at posterior wall of stomach. This is facilitated by transection of greater omentum and exposure of lesser sac.

Resection of tumor with linear stapler is sometimes performed for pedunculated gastric wall tumor with extragastric extension. This minimizes the risk of tumor seeding and peritoneal contamination by gastric content. However, this is not suitable for tumor with intra-gastric extension or involvement of a wide area of gastric wall. Otherwise, incomplete resection of tumor may result or more extensive 
resection of gastric wall adjacent to the tumor is inevitable. Furthermore, resection by linear stapler is not suitable for tumor located along gastric lesser curve or close to cardia and pylorus.

Gastrostomy followed by resection of gastric tumor with clear margin was performed in our patient due to the wide area of gastric wall involvement. Ultrasonic dissector, with good hemostatic performance for gastric wall vessels, is used to resect localized gastric wall tumor. Careful and gentle manipulation of tumor during dissection should be practiced to avoid breakage of tumor capsule and rupture of the tumor. Immediate placement of tumor in a plastic specimen bag and adequate peritoneal lavage were performed to reduce risk of tumor seeding.

Suture repair of gastrostomy can be difficult without good exposure and close cooperation between the chief surgeon and assistant. Omental fat next to the edge of gastrostomy should be dissected free to facilitate proper placement of sutures. Gentle pull of the suture material is very important especially when the suture repair is performed in a continuous manner.

\section{Acknowledgements}

None.

\section{Footnote}

Conflicts of Interest: The authors have no conflicts of interest to declare.

\section{References}

1. Catena F, Di Battista M, Fusaroli P, et al. Laparoscopic treatment of gastric GIST: report of 21 cases and literature's review. J Gastrointest Surg 2008;12:561-8.

doi: 10.21037/jovs.2016.06.02

Cite this article as: Yang X, Jiang L, Chan FS. Laparoscopic resection of gastric wall tumor. J Vis Surg 2016;2:116.
2. Bédard EL, Mamazza J, Schlachta CM, et al. Laparoscopic resection of gastrointestinal stromal tumors: not all tumors are created equal. Surg Endosc 2006;20:500-3.

3. Choi SM, Kim MC, Jung GJ, et al. Laparoscopic wedge resection for gastric GIST: long-term follow-up results. Eur J Surg Oncol 2007;33:444-7.

4. Huguet KL, Rush RM Jr, Tessier DJ, et al. Laparoscopic gastric gastrointestinal stromal tumor resection: the mayo clinic experience. Arch Surg 2008;143:587-90; discussion 591.

5. Lai IR, Lee WJ, Yu SC. Minimally invasive surgery for gastric stromal cell tumors: intermediate follow-up results. J Gastrointest Surg 2006;10:563-6.

6. Nguyen SQ, Divino CM, Wang JL, et al. Laparoscopic management of gastrointestinal stromal tumors. Surg Endosc 2006;20:713-6.

7. Novitsky YW, Kercher KW, Sing RF, et al. Longterm outcomes of laparoscopic resection of gastric gastrointestinal stromal tumors. Ann Surg 2006;243:73845; discussion 745-7.

8. Rivera RE, Eagon JC, Soper NJ, et al. Experience with laparoscopic gastric resection: results and outcomes for 37 cases. Surg Endosc 2005;19:1622-6.

9. Pelletier JS, Gill RS, Gazala S, et al. A Systematic Review and Meta-Analysis of Open vs. Laparoscopic Resection of Gastric Gastrointestinal Stromal Tumors. J Clin Med Res 2015;7:289-96.

10. Demetri GD, Benjamin RS, Blanke CD, et al. NCCN Task Force report: management of patients with gastrointestinal stromal tumor (GIST)--update of the NCCN clinical practice guidelines. J Natl Compr Canc Netw 2007;5 Suppl 2:S1-29; quiz S30.

11. Yang X, Jiang L, Chan FS. Laparoscopic resection of gastric wall tumor. Asvide 2016;3:272. Available online: http://www.asvide.com/articles/1034 